\title{
Ion and electron heating at the Martian bow shock. Common for bow shocks or not?
}

\author{
A. Skalsky ${ }^{1,5}$, E. M. Dubinin ${ }^{1,2,5}$, A. Petrukovich ${ }^{1}$, K. Sauer ${ }^{2,5}$, P. Kiraly ${ }^{3}$, and K. Schwingenschuh ${ }^{4}$ \\ ${ }^{1}$ Space Research Institute, Profsoyuznaya 84/32, 117810, GSP-7 Moscow, Russia \\ ${ }^{2}$ Max-Planck-Institut für Aeronomie, D-37191 Katlenburg-Lindau, Germany \\ ${ }^{3}$ Central Research Institute for Physics, H-1525 Budapest, P.O. Box 49, Hungary \\ ${ }^{4}$ Institut fur Weltraumforschung/OAW, $8010 \mathrm{Graz}$, Inffeldgasse 12, Austria \\ ${ }^{5}$ International Space Science Institute (ISSI), Hallerstrasse 6, 3012 Bern, Switzerland
}

(Received August 4, 1997; Revised January 19, 1998; Accepted January 28, 1998)

\begin{abstract}
Two typical bow shock crossings recorded by the Phobos- 2 spacecraft in 1989 are considered in the present paper in order to demonstrate that the Martian bow shock is the shock of "common sense" in spite of peculiarities due to the pick-up ions of the Martian origin and their Larmour radius comparable to the scale size of the interaction region between the planet and solar wind. The incident plasma flow is decelerated and plasma species are heated within the relatively thin layer upstream the planet. The observed changes of plasma density, velocity and temperature are comparable with values expected for the MHD shock waves. Moreover, the dynamics of ion and electron energy distributions observed in the shock transition region indicates that mechanisms responsible for the energy dissipation seems to be similar to those operating at the Earth's bow shock.
\end{abstract}

\section{Introduction}

The plasma and magnetic field measurements performed by the Phobos-2 spacecraft have confirmed previous observations made on Mars-3, 5 and Mariner-4 that the bow shock exists near Mars (Riedler et al., 1989; Schwingenschuh et al., 1990). Barabash and Lundin (1993) have distinctly observed a foot of reflected protons at the subsolar bow shock, and evaluated that about $30 \%$ of solar wind protons might be reflected at the electrostatic barrier of the shock. Trotignon et al. (1991) have compared spectra of plasma waves at Earth's and Mars's bow shocks that were measured by similar instruments onboard Phobos- 1 and Phobos- 2 spacecraft. The similar features of electric-field amplitude spectra were found. Tatrallyay et al. (1997) have analyzed magnetic field overshoots in the terminator Martian bow shock and found features which are similar to those observed at Earth and Venus. The height of overshoot increased with the Mach number, and the thickness was typically $0.5-2.5$ proton gyroradii. Barabash and Lundin (1993) have identified the well developed ion foreshock upstream of the Martian bow shock which was very similar to that known from observations around the Earth. Ions reflected from the bow shock and streaming back to the solar wind generate ULF waves which resemble those reported for the Earth foreshock (Delva and Dubinin, 1998). The wave measurements performed by the Plasma Wave System (PWS) allowed to identify the electron foreshock at Mars (Skalsky et al., 1992). Skalsky et al. (1992) reported observations of the emissions at frequencies around the local electron plasma frequency after the spacecraft crossed the magnetic field line tangential to the bow shock surface. These high

Copy right(C) The Society of Geomagnetism and Earth, Planetary and Space Sciences (SGEPSS); The Seismological Society of Japan; The Volcanological Society of Japan; The Geodetic Society of Japan; The Japanese Society for Planetary Sciences. frequency waves, previously discovered in the near Earth's space, are generated by electrons reflected at the bow shock (Feldman et al., 1983, Fitzenreiter et al., 1990). Simultaneous measurements of electrons at Mars revealed also the enhanced level of particles with energies between 100 and $530 \mathrm{eV}$ reflecting from the Martian bow shock (Skalsky et al., 1993). All these observational facts provide an evidence that physical processes ongoing at the Martian bow shock and in its upstream region are very similar to those known for the Earth environment.

On the other hand, some new features of the Martian bow shock were also found. Because the shock is immersed an extended neutral exosphere, a new population of ions may be originated in front of shock. Barabash et al. (1991) observed pickup protons outside the foreshock. Russell et al. (1990) measured wave emissions at proton gyrofrequency and attributed them to pick-up ions. Delva and Dubinin (1998) found ULF fluctuations of the magnetic field upstream of the foreshock. Dubinin et al. (1993, 1995) showed that pickedup exospheric protons reflected from the bow shock contribute significantly to the population of backstreaming ions. Moses et al. (1989) pointed out another interesting feature of the Martian bow shock. Because of the large gyroradius of reflected solar wind protons, a partial overlapping of quasiparallel and quasiperpendicular shocks may happens. Moreover, small scale of Mars gives rise to doubts about the existence of the traditional collisionless bow shock. Brecht and Ferrante (1991) and Brecht (1997) argued that ion dissipation caused by ion reflection does not exist around Mars, because of the lack of room. Solar wind gyroradius $1200-2900 \mathrm{~km}$ is comparable with a scale of obstacle $\left(R_{\mathrm{M}} \sim 3380 \mathrm{~km}\right)$ and could exceed the thickness of the magnetosheath at subsolar region. Based on 3-D hybrid simulations on interaction be- 
PHOBOS-2, 22 MARCH 1989


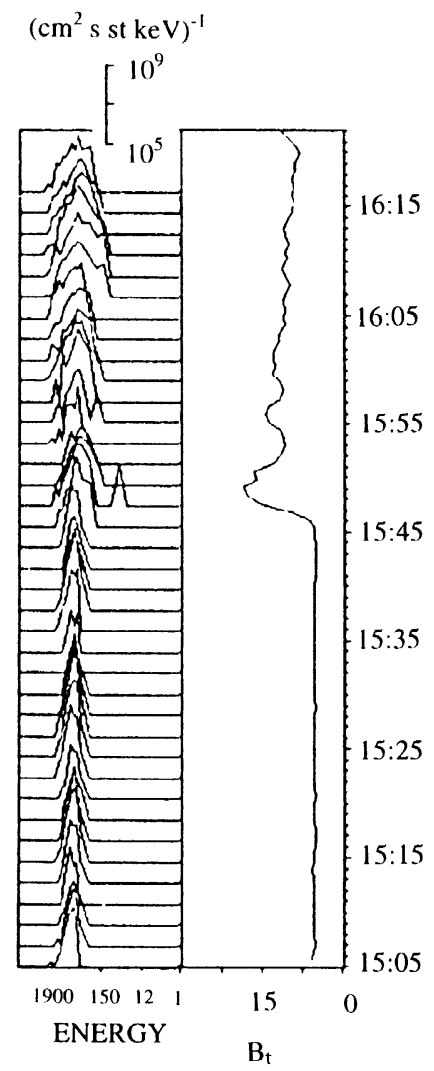

Fig. 1. Proton observations made with the ASPERA instrument on 22 March 1989 when the Martian bow shock is crossed. Energy-time spectrogram of proton fluxes along with the magnetic field strength are presented at the left side; proton temperatures, proton velocities and magnetic field value are shown from top to bottom at the right side.

tween the solar wind and Mars, Brecht (1997) concluded the difference of 'the kinetic wave-like behavior of the Martian bow shock and standard hydrodynamic paradigm'. The main topics of the paper are to present two typical crossings of the Martian bow shock and to consider the dynamics of electrons and ions through the shock transition region at Mars with an emphasize on how they agree with expectations for the MHD shock and observations at the Earth's bow shock.

\section{Ion Heating}

The ASPERA ion spectrometer onboard the Phobos-2 spacecraft, comprised the toroidal $(E / q)$ analyzer placed in front of cross-field mass separator, was able to measure energy and mass distribution of ions. A set of ten detectors was used to measure energy spectra $(0.5 \mathrm{eV} / q-25 \mathrm{keV} / q)$ of ions coming from 10 directions. The field of view of the spectrometer system was $5^{\circ} \times 360^{\circ}$. The absence of reliable attitude measurements significantly entangled the 'despinning procedure' of onboard moment calculations (Kallio et al., 1994). To avoid this problem, only 'spectral' data of the ASPERA were used to evaluate fluid parameters of protons. $E / q$-spectra of protons were measured every $1 \mathrm{~min}$ from sunward, antisunward and side directions. Figure 1 presents observations of the solar wind protons carried out with the ASPERA during the inbound crossing of the Martian bow shock at 15:47 UT on 22 March 1989. At this time, the Phobos-2 spacecraft was in the evening sector at the distance of $\sim 2.8 R_{\mathrm{M}}$ from the center of the planet; the zenith angle was about $90^{\circ}$. The solar wind conditions on 22 March lead to the following 'shock' parameters: $M_{\mathrm{A}} \sim 5.9$, $M_{\mathrm{MS}} \sim 5.5, \beta_{\mathrm{i}} \sim 0.25, \beta_{\mathrm{e}} \sim 0.35$. The observed profile of the magnetic field magnitude shown in Fig. 1 is typical for the quasiperpendicular shock. The foot region can be identified between 15:45 UT and 15:47 UT; the shock ramp is encountered at 15:47 UT. The solar wind protons are mainly heated at the shock ramp simultaneously with the sharp deceleration of the solar wind plasma (Fig. 1). The gyrating ions with energies higher than those of the undisturbed solar wind are observed in the downstream between 15:47 and 16:05 UT. Those ions were reflected from the bow shock and gained energy in $\mathbf{V} \times \mathbf{B}$ field. Then, they passed through the shock and contributed to the further ion heating. The temperature of protons slightly increases at 16:05 UT and the high-energy tail appears at energies at which the gyrating ions are observed earlier. Dubinin et al. (1993) considered this bow shock encounter with an emphasize on how the exospheric ions can influence the physical processes ongoing at the Martian bow shock. The ions at low energies, recorded by another detector and interpreted as those of the Martian origin, appear near the shock front with an increase at about 16:05 UT, i.e. after the solar wind ions have been heated in the shock transition region. Thus, the Martian ions are mostly affected the solar wind flow in the depth of the magnetosheath. Ion kinetic parameters presented in Fig. 1 (left 
PHOBOS-2 1 March 1989

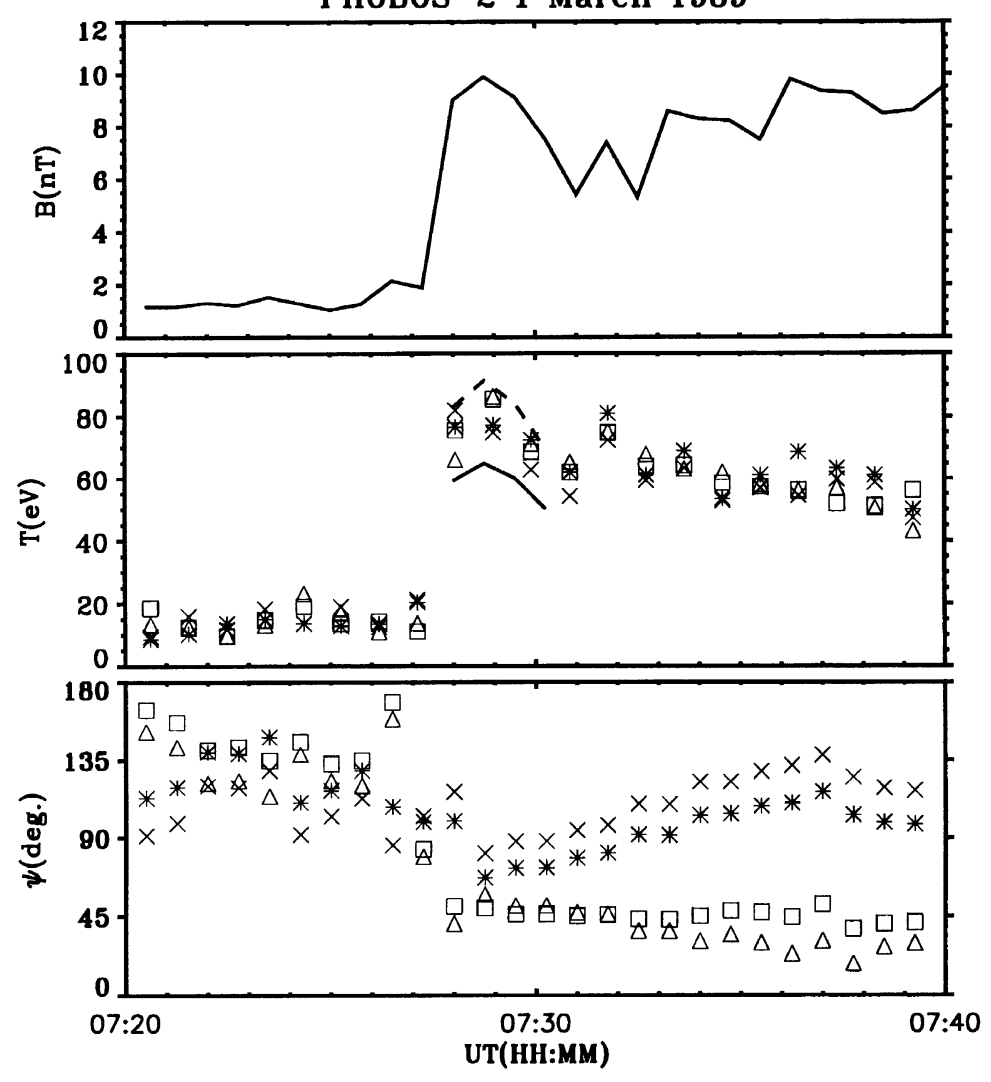

Fig. 2. A bow shock crossing on 1 March 1989. The magnetic field magnitude, the temperatures calculated with measurements of four slits of the HARP instrument (namely, those making angles of $-56^{\circ}$ (squares), $-33^{\circ}$ (triangles), $33^{\circ}$ (stars) and $56^{\circ}$ (crosses) with the anti-solar direction) and the respective pitch-angles $\psi$ of electrons entering these slits are shown from top to bottom. The solid and dashed lines in the middle panel indicate the limits of the electron heating under the assumption of $T_{\mathrm{e} \perp} / B$ conservation (see explanation in the text).

panel) are obtained with the moment calculations. The ratio of ion heating $\left(T_{\mathrm{d}} / T_{\mathrm{u}}\right.$, the subscript " $\mathrm{u}$ " denotes upstream values; the subscript "d" is used for downstream values) is about 18 for the shock crossing presented. The value of ion temperature in the downstream region, which is used to estimate the ratio of heating, is taken after 16:05 UT when the relaxation of gyrating ions completes. The value of 18 for the heating ratio of ions is rather typical for observation at the Martian bow shock. The part of the energy dissipated in the shock which transforms into the thermal energy of ions is about $20 \%$. The jump of the magnetic field $B_{\mathrm{d}} / B_{\mathrm{u}}$ is about of factor 2 if the value of $B_{\mathrm{d}}$ is taken after the relaxation of the gyrating ions, and does not exceed a factor of 3 for $B_{\mathrm{d}}$ taken in the overshoot at 15:48 UT. It implies that the ion heating is strongly non-adiabatic, i.e. its ratio significantly exceeds the jump in the magnetic field value at the shock front.

\section{Electron Heating}

The hyperbolic electrostatic analyzer HARP have measured the energy spectra of electrons in eight viewing sectors arranged in a fan configuration with the symmetry axis pointing in the anti-solar direction. The plane of the fan was perpendicular to the ecliptic plane when the spacecraft was in a three-axis stabilized mode. Each slit covered a field of view of $10^{\circ} \times 20^{\circ}$ within and across the fan plane, respectively. The electron spectra were measured in 25 energy steps in the range from 3.4 to $550 \mathrm{eV}$. The inbound bow shock crossing on 1 March 1989 was detected at 07:28 UT. Data on the solar wind conditions on 1 March 1989 lead to the following plasma parameters: $M_{\mathrm{A}} \sim 21, M_{\mathrm{MS}} \sim 6, \beta_{\mathrm{i}} \sim 1.1$ and $\beta_{\mathrm{e}} \sim 3.5$. The complanarity theorem and the model of the shock shape (Trotignon et al., 1991) are used to derive the angle $\varphi$ between the normal to the shock and the magnetic field in the solar wind. The angle, estimated with both methods, is between $25^{\circ}$ and $40^{\circ}$. However, the increase of the magnetic field magnitude after 07:33 UT (Fig. 2) seems to depend on the variation of the magnetic field in the solar wind. It makes the evaluation of the angle somewhat uncertain. Nevertheless, the profile of the magnetic field allows to consider at least, this bow shock crossing as the intermediate one $\left(\varphi=45^{\circ}\right)$. Figure 2 presents, from top to bottom, the magnetic field magnitude $B$, the electron temperatures $T_{\mathrm{e}}$ measured with different slits of the HARP instrument (namely, those making angles of $-56,-33,33$ and $56^{\circ}$ with the antisolar direction) and the pitch-angles $\psi$ of electrons entering these slits. The electron temperatures were calculated with algorithms described by Montgomery et al. (1970) and Scudder et al. (1973). The electron temperature upstream of the shock front was obtained by fitting the measured distributions with a Maxwellian function. The Maxwellian shape of the electron distribution function in the solar wind was 
proved by many authors (see, for example, Feldman et al. (1975) and Rosenbauer et al. (1976)). The similar conclusion with regard to the electron measurements in the solar wind near Mars has been delivered by Shutte et al. (1991). The measure of temperature in the magnetosheath was evaluated by calculating the second moment of the electron distribution function in view of its non-Maxwellian form. The electron flux at energies below $25 \mathrm{eV}$ was probably affected by the spacecraft potential and measurements in this energy range were excluded from calculations.

Figure 2 shows that the electron heating occurs in the thin region around the shock front simultaneously with a sharp increase in the magnetic field magnitude. The curve segments shown in the middle panel has been evaluated with the following relations: $T_{\mathrm{eu}}\left(B_{\mathrm{d}} / B_{\mathrm{u}}\right)$ (dashed line) and ( $\left.2 T_{\mathrm{eu}} B_{\mathrm{d}} / B_{\mathrm{u}}+T_{\mathrm{eu}}\right) / 3$ (solid line) where $T_{\mathrm{e}}$ is the electron temperature and $B$ is the magnetic field value. The subscripts "u" and "d" denote parameters upstream and downstream the shock front respectively. The first relation defines the $\Delta T_{\mathrm{e} \perp}$ with the assumption of the $T_{\mathrm{e} \perp} / B=$ const. The second re-

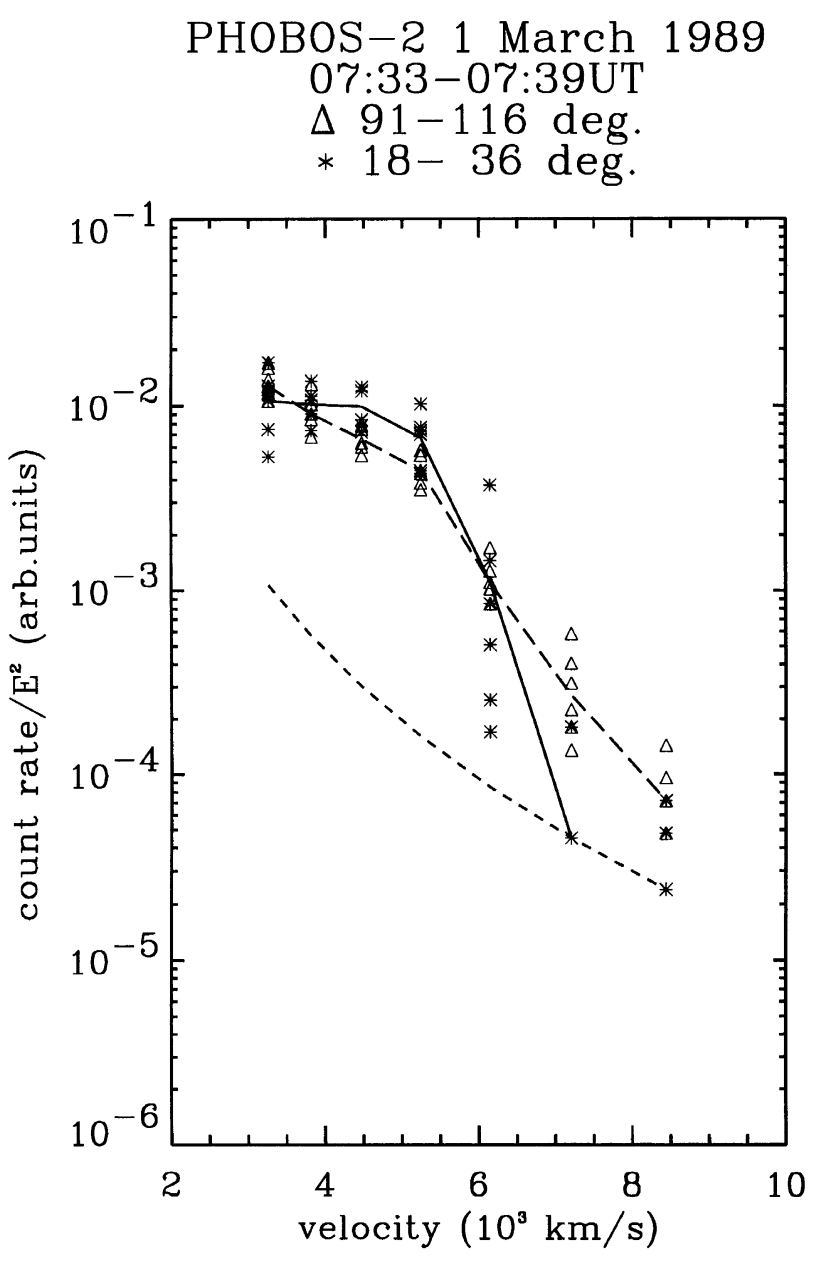

Fig. 3. Electron velocity distributions observed with two slits of the HARP spectrometer in the magnetosheath in the time interval 07:33-07:39 UT on 1 March 1989. The pitch-angles intervals in which distributions are detected are indicated at the top. The median shapes of the electron velocity distributions in the pitch-angle intervals $91-116^{\circ}$ and $18-36^{\circ}$ are shown with dashed and solid lines respectively. The reference level corresponding to 1 count/sec is shown with a small dashes. lation gives the estimate of the temperature increase under the following assumptions: $T_{\mathrm{eu} \perp}=T_{\mathrm{eu} \|}$; an absence of any heating parallel to the magnetic field and a certain redistribution of the electron energy in the pitch angles downstream the shock front is due to, for example, the magnetic field turbulence (Schwartz et al., 1988). Behind the shock front, the values of temperature derived from observations are in the margins defined with these two relations which leads to the conclusion that the observed increase in the electron temperature is mostly adiabatic at the shock front.

Figure 3 presents the compilation of electron velocity distribution functions measured by two slits of the HARP instrument in the magnetosheath throughout the time interval 07:33-07:39 UT on 1 March 1989. The two slits, covering the pitch angles closest to $0^{\circ}$ and $90^{\circ}$, are chosen and median shapes are presented with solid and dashed lines respectively. It is seen that the electron distribution measured closer to the magnetic field reveals the flat-top shape at low energies with a break in slope at the energy of about $60 \mathrm{eV}\left(4.6 \cdot 10^{3} \mathrm{~km} / \mathrm{s}\right)$. The electron distribution in the pitch-angle range $91-116^{\circ}$ is less flat at low energies and, beyond $60 \mathrm{eV}\left(4.6 \cdot 10^{3} \mathrm{~km} / \mathrm{s}\right)$, its slope is less steep than that of the spectrum measured closest to the magnetic field direction.

\section{Discussion and Conclusion}

Earlier studies on the ion dynamics at the Earth's bow shock point out that the following features are commonly observed for both critical and supercritical shocks (Thomsen et al., 1985; Sckopke et al., 1990 and references in these papers): (1) ions reflected from the shock rump and accelerated by the solar wind electric field in the foot region; (2) sharp broadening of ion distribution (heating) at the shock rump; (3) gyrating ions in the downstream region; (4) their relaxation leading to the high energy non-Maxwellian tail in the depth of downstream region; (5) the observed ratio of proton heating exceeds the adiabatic level. These phenomena typically observed in the near-Earth space resemble those presented in this paper. Moreover, the proton heating ratio at Mars, which is typically of 18 , can be compared to that of 16 usually observed at the Earth's bow shock with similar Mach numbers (Formisano et al., 1973a,b). For both planetary shocks, the part of energy of incoming solar wind which is converted to the thermal motion of protons is within $20 \%$. Further, Formisano et al. $(1973 \mathrm{a}, \mathrm{b})$ have also shown that the plasma behavior at the Earth's bow shock, particularly jumps of plasma densities, velocities, temperatures, fraction of dissipated energy follow expectations for the MHD shock waves. The resemblance between ion dynamics at Martian and terrestrial bow shocks allows to conclude that MHD theory is generally applicable for the Martian bow shock, at least, at the terminator region. Similar conclusion was made by Vaisberg et al. (1990) who compared the observed jumps of the proton temperature with values predicted by the MHD theory. Study, based on ion data from the Mars-2, 3, 5 spacecrafts shows that although the observed jumps are less than expected (an electron temperature is not included in calculations), the solar wind is deflected by the strong bow shock without significant absorption of the flow by planet. The bow shock encounter on 22 March 1989 allows to demonstrate the most typical features of ion dynamics observed 
in the shock transition region near the terminator of Mars. Ion deceleration and heating inside the shock transition are not strongly influenced by exospheric ions. The "cold" ions reported by Dubinin et al. (1993) are 'mixing' in velocity space with the solar wind protons deeply inside the magnetosheath when the solar wind ions are already heated (see, for example Fig. 3 from Dubinin et al., (1993)). A total thermalization affecting the whole community occurs over the magnetosheath and plasma mantle. However, there is a limited set of observations, particularly near the subsolar point, when the solar wind ions reveal the unusual dynamics at the shock front. These events are associated with the enhanced fluxes of the exospheric ions, large Mach numbers and ion gyroradius (Dubinin et al.,1994). The full ion kinetic treatment performed by Brecht and Ferrante (1991) and Brecht (1997) under the solar wind conditions similar to those observed during these unusual shock crossings indicates the absence of the pronounced overshoot in the quasi-perpendicular subsolar region. Even so, in-situ ion measurements carried out near the subsolar point reveal the relatively sharp change of the solar wind flow kinetic parameters which allows to state the crossing of the bow shock (Lundin et al., 1989).

The electron heating at the oblique Martian bow shock encountered near the terminator occurs in the thin layer around the shock ramp and is mostly adiabatic. This result can be compared with observation at the Earth's bow shock where the electron heating is ongoing at the shock front being also adiabatic for the most part of quasiperpendicular crossings with different Mach numbers considered by Schwartz et al. (1988) and Thomsen et al. (1985). The spectra shapes measured downstream the front of the Martian bow shock are obviously reminiscences of those in the the Earth's magnetosheath reported by Montgomery et al. (1970), Scudder et al. (1973) and, by Feldman et al. (1983). Indeed, electron distribution in the earth magnetosheath has a flat top at energies below, roughly $100 \mathrm{eV}$ which is the most evident along the magnetic field. The electron distribution function diminishes and the slope measured perpendicular to the magnetic field is less steep than that of distribution along the magnetic field above this energy. Both features are quite similar to those presented in this paper. Unfortunately, the data base of the electron observations in the vicinity of the Martian bow shock is limited to three sequences recorded when the HARP instrument have been operated with the relatively high telemetry rate. Two other records of electron data measured at the Martian bow shock and described by Shutte et al. (1991) and Kiraly et al. (1991) were collected during crossings of the quasiperpendicular region of the planetary bow shock, near the subsolar point on 1 and 4 February 1989. The electron flux exhibits maximum values at energies of about or higher $530 \mathrm{eV}$ downstream the shock front in both cases $(530 \mathrm{eV}$ is the upper energy limit of the HARP instrument). This fact implies a very strong heating of electrons which exceeds the adiabatic level provided by the jump of the magnetic field strength (Schwingenschuh et al., 1990). However, it could be explained by rather high solar wind with bulk velocities of $790 \mathrm{~km} / \mathrm{sec}$ and $550 \mathrm{~km} / \mathrm{sec}$ on 1 February 1989 and 4 February 1989, respectively. Indeed, a few observations made in the near-Earth under the high-speed conditions in the solar wind $\left(V_{\mathrm{sw}}>550 \mathrm{~km} / \mathrm{sec}\right)$ reveal also very large increase of the electron temperature $\left(T_{\mathrm{d}} / T_{\mathrm{u}}>10\right)$ at the bow shock which exceeds the jump of the magnetic field (Thomsen et al., 1987). In spite of poor statistics of electron observations onboard the Phobos-2 spacecraft, general similarities between the electron behavior at Earth and Mars can be stated. In particular, it is related to the mechanism of electron thermalization. It appears that the electron heating is the same in nature for both planetary shocks and is governed in the same way by the solar wind conditions.

The planetary environment at Mars has certain features, particularly pick-up protons and cold ionospheric ions, which makes it different from that of the Earth (Dubinin et al., 1993, 1995; Barabash et al., 1991). Even so, the ion and electron dynamics in the vicinity of two planetary bow shocks are very similar. It leads to the conclusion that the Martian bow shock is indeed the "shock". Moreover, the microscopic processes responsible for the energy dissipation, ion and electron reflections in the shock region at Mars are believed to be similar to those operating at the Earth's bow shock.

Acknowledgments. The authors wish to express their thanks to the ISSI in Bern for the support in the collaboration in the framework of Visiting Science Program. The work of A. Skalsky was also supported by the INTAS grant 94-3626.

\section{References}

Barabash, S. and R. Lundin, Reflected ions near Mars, Phobos-2 observations, Geophys. Res. Lett., 20, 787-790, 1993.

Barabash, S., E. Dubinin, N. Pissarenko, and C. T. Russell, Picked-up protons near Mars: Phobos-observations, Geophys. Res. Lett., 18, 1805 1808, 1991.

Brecht, S. H., Hybrid simulations of the magnetic topology of Mars, $J$. Geophys. Res., 102, 4743-4750, 1997.

Brecht, S. H. and J. R. Ferrante, Global hybrid simulation of unmagnetized planets: comparison of Venus and Mars, J. Geophys. Res., 96, 1120911220, 1991.

Delva, M. and E. Dubinin, Upstream ULF fluctuations near Mars, J. Geophys. Res., 103, 317-326, 1998.

Dubinin, E., R. Lundin, H. Koskinen, and O. Norberg, Cold Ions at the Martian bow shock: Phobos Observations, J. Geophys. Res., 98, 56175623, 1993

Dubinin, E., D. Obod, A. Pedersen, and R. Grard, Mass-loading asymmetry in the upstream region near Mars, Geophys. Res. Lett., 21, 2769-2772, 1994

Dubinin, E., D. Obod, R. Lundin, K. Schwingenschuh, and R. Grard, Some features of the martian bow shock, Adv. Space Res., 15, 423-431, 1995.

Feldman, W. C., J. R. Asbridge, S. J. Bame, M. D. Montgomery, and S. P Gary, Solar wind electrons, J. Geophys. Res., 80, 4181-4196, 1975.

Feldman, W. C., R. C. Anderson, S. J. Bame, S. P. Gary, J. T. Gosling, D. J. McComas, M. F. Thomsen, G. Paschmann, and M. M. Hoppe, Electron velocity destribution near the Earth's bow shock, J. Geophys. Res., 88, 96-110, 1983.

Fitzenreiter, R. J., J. D. Scudder, and A. J. Klimas, Three dimensional analytical model for the spatial variation of the foreshock distribution function: systematic and comparisons with ISEE observation, J. Geophys. Res., 95, 4155-4173, 1990.

Formisano, V., G. Moreno, F. Palmiotto, and P. C. Hedgecock, Solar wind interaction with the Earth's magnetic field, 1. Magnetosheath, J. Geophys. Res., 78, 3714-3730, 1973a.

Formisano, V., P. C. Hedgecock, G. Moreno, F. Palmiotto, and J. K. Chao, Solar wind interaction with the Earth's magnetic field, 2. Magnetohydrodynamic Bow Shock, J. Geophys. Res., 78, 3731-3760, 1973b.

Kallio, E., H. Koskinen, S. Barabash, R. Lundin, O. Norberg, and J. G. Luhmann, Proton flow in the Martian magnetosheath, J. Geophys. Res., 99, 23547-23559, 1994.

Kiraly, P. et al., The HARP plasma experiment on-board the PHOBOS-2 spacecraft: preliminary results, Planet. Space Sci., 39, No.1/2, 139-145, 1991.

Lundin, R., A. Zakharov, R. Pellinen, H. Borg, B. Hultqvist, N. Pissarenko, E. Dubinin, S. Barabash, I. Liede, and H. Koskinen, First measurements 
of the ionospheric plasma escape from Mars, Nature, 341, 609-612, 1989.

Montgomery, M. D., J. R. Asbridge, and S. J. Bame, Vela 4 plasma observations near the Earth's bow shock, J. Geophys. Res., 75, 1217-1235, 1970 .

Moses, S. L., F. V. Coronoti, and F. L. Scarf, Expectations for the microphysics of the Mars-solar wind interaction, Geophys. Res. Lett., 15, 429-432, 1989.

Riedler, W. et al., Magnetic fields near Mars: first results, Nature, 341, 604-607, 1989.

Rosenbauer, H., H. Miggenrieder, M. Montgomery, and R. Schwenn, Preliminary results of the Helios plasma measurements, in Physics of Solar Planetary Environments, vol. 1, edited by D. J. Williams, p. 319, AGU, 1976.

Russell, C. T., J. G. Luhmann, K. Scwingenschuh, W. Riedler, and Ye. Yeroshenko, Upstream waves at Mars: Phobos observations, Geophys. Res. Lett., 17, 897-900, 1990.

Schwartz, S. J., M. F. Thomsen, S. J. Bame, and J. Stansberry, Electron heating and the potential jump across fast mode shock, J. Geophys. Res., 93, 12923-12931, 1988.

Schwingenschuh, K., W. Riedler, H. Lichtenegger, Ye. Yeroshenko, K. Sauer, J. G. Luhmann, M. Ong, and C. T. Russell, Martian bow shock: Phobos observations, Geophys. Res. Lett., 17, 889-892, 1990.

Sckopke, N., G. Paschman, A. L. Brinca, C. W. Carlson, and H. Luhr, Ion thermalization in quasiperpendicular shocks involving reflected ions, $J$. Geophys. Res., 95, 6337-6352, 1990

Scudder, J. D., D. L. Lind, and K. W. Ogilvie, Electron observations in the solar wind and magnetosheath, J. Geophys. Res., 78, 6535-6548, 1973.

Shutte, N. M. et al., Energy distribution of electrons with $E<800 \mathrm{eV}$ in the areomagnetosphere, Planet. Space Sci., 39, No. 1/2, 147-151, 1991.
Skalsky, A., R. Grard, C. Nairn, S. Klimov, J.-G. Trotignon, and K. Schwingenschuh, The Martian bow shock: wave observations in the upstream region, J. Geophys. Res., 76, 2927-2933, 1992.

Skalsky, A., R. Grard, P. Kiraly, S. Klimov, V. Kopanyi, K. Scwingenschuh, and J. G. Trotignon, Simultaneous plasma waves and electron flux observations upstream of the Martian bow shock., Planet. Space Sci., 41, 183-188, 1993.

Tatrallyay, M., G. Gevai, I. Apathy, K. Schwingenschuh, T-L. Zhang, G. Kotova, M. Verigin, S. Livi, and H. Rosenbauer, Magnetic field overshoots in the Martian bow shock, J. Geophys. Res., 102, 2157-2163, 1997.

Thomsen, M. F., J. T. Gosling, and S. J. Bame, Ion and electron heating at collisionless shocks near the critical Mach number, J. Geophys. Res., 90, 137-148, 1985.

Thomsen, M. F., M. M. Mellott, J. A. Stansberry, S. J. Bame, J. T. Gosling, and C. T. Russell, Strong electron heating at the Earth's bow shock, $J$. Geophys. Res., 92, 10119-10124, 1987.

Trotignon, J. G., M. Hamelin, R. Grard, A. Pedersen, S. Klimov, S. Savin, A. Skalsky, and C. Kennel, A comparison between the Earth's and Mars' bow shocks detected by the PHOBOS plasma wave system, Planet. Space Sci., 39, 99-112, 1991.

Vaisberg, O. L., J. G. Luhmann, and C. T. Russell, Plasma observations of the solar wind interaction with Mars, J. Geophys. Res., 95, 14841-14852, 1990 .

A. Skalsky (e-mail: askalsky@vm1.iki.issi.ru), E. M. Dubinin (e-mail: dubinin@helene.mpae.gwdg.de), A. Petrukovich, K. Sauer (e-mail: sauer@ linax1.mpae.gwdg.de), P. Kiraly, and K. Schwingenschuh 